\title{
One-Year Brain Atrophy Evident in Healthy Aging
}

\author{
Anders M. Fjell, ${ }^{1}$ Kristine B. Walhovd, ${ }^{1}$ Christine Fennema-Notestine, ${ }^{2,3}$ Linda K. McEvoy, ${ }^{2}$ Donald J. Hagler, ${ }^{2}$ \\ Dominic Holland, ${ }^{4}$ James B. Brewer, ${ }^{2,4}$ and Anders M. Dale ${ }^{2,4}$ \\ ${ }^{1}$ Center for the Study of Human Cognition, Department of Psychology, University of Oslo, 0317 Oslo, Norway, ${ }^{2}$ Department of Radiology, University of \\ California, San Diego, La Jolla, California 92103, and Departments of ${ }^{3}$ Psychiatry and ${ }^{4}$ Neurosciences, University of California, San Diego, La Jolla, \\ California 92093
}

An accurate description of changes in the brain in healthy aging is needed to understand the basis of age-related changes in cognitive function. Cross-sectional magnetic resonance imaging (MRI) studies suggest thinning of the cerebral cortex, volumetric reductions of most subcortical structures, and ventricular expansion. However, there is a paucity of detailed longitudinal studies to support the cross-sectional findings. In the present study, 142 healthy elderly participants (60-91 years of age) were followed with repeated MRI, and were compared with 122 patients with mild to moderate Alzheimer's disease (AD). Volume changes were measured across the entire cortex and in 48 regions of interest. Cortical reductions in the healthy elderly were extensive after only 1 year, especially evident in temporal and prefrontal cortices, where annual decline was $\sim 0.5 \%$. All subcortical and ventricular regions except caudate nucleus and the fourth ventricle changed significantly over 1 year. Some of the atrophy occurred in areas vulnerable to $\mathrm{AD}$, while other changes were observed in areas less characteristic of the disease in early stages. This suggests that the changes are not primarily driven by degenerative processes associated with $\mathrm{AD}$, although it is likely that preclinical changes associated with $\mathrm{AD}$ are superposed on changes due to normal aging in some subjects, especially in the temporal lobes. Finally, atrophy was found to accelerate with increasing age, and this was especially prominent in areas vulnerable to AD. Thus, it is possible that the accelerating atrophy with increasing age is due to preclinical AD.

\section{Introduction}

An accurate description of age-related changes in the brain is needed to better understand the neurobiological foundation of cognitive changes in healthy aging. While cross-sectional magnetic resonance imaging (MRI) studies have demonstrated consistent age-effects (Raz et al., 2004; Salat et al., 2004; Allen et al., 2005; Walhovd et al., 2005; Raz and Rodrigue, 2006; Fjell et al., 2009a,b; Westlye et al., 2009), longitudinal MR studies have

\footnotetext{
Received July 8, 2009; revised 0ct. 15, 2009; accepted 0ct. 23, 2009.

This work was supported by a grant (U24 RR021382) to the Morphometry Biomedical Informatics Research Network (http://www.nbirn.net) that is funded by the National Center for Research Resources at the National Institutes of Health (NIH), and by the National Institute on Aging at the NIH (U01 AG024904, R01 AG031224, and K01AG029218). The work of K.B.W. and A.M.F. was supported by the Norwegian Research Council. Data collection and sharing for this project were funded by the Alzheimer's Disease Neuroimaging Initiative (ADNI; Principal Investigator: Michael Weiner; NIH Grant U01 AG024904). ADNI is funded by the National Institute on Aging, the National Institute of Biomedical Imaging and Bioengineering, and generous contributions from the following: Pfizer, Wyeth Research, Bristol-Myers Squibb, Eli Lilly and Company, GlaxoSmithKline, Merck, AstraZeneca, Novartis Pharmaceuticals, Alzheimer's Association, Eisai Global Clinical Development, Elan, Forest Laboratories, and the Institute for the Study of Aging, with participation from the United States Food and Drug Administration. Industry partnerships are coordinated through the Foundation for the National Institutes of Health. The grantee organization is the Northern California Institute for Research and Education, and the study was coordinated by the Alzheimer's Disease Cooperative Study at the University of California, San Diego. ADNI data are disseminated by the Laboratory of Neuro Imaging at the University of California, Los Angeles. We thank Robin Jennings, Michele Perry, Chris Pung, and Elaine Wu for downloading and preprocessing the ADNI MRI data. Data used in the preparation of this article were obtained from the ADNI database (www.loni.ucla.edu/ADNI). As such, the investigators within the ADNI contributed to the design and implementation of ADNI and/or provided data but did not participate in analysis or writing of this report. A complete listing of ADNI investigators is available at http://www.loni.ucla.edu/ADNI/Data/ ADNI_Authorship_List.pdf.

Correspondence should be addressed to Anders M. Fjell, Department of Psychology, University of 0slo, P.0. Box 1094 Blindern, 0317 0slo, Norway. E-mail: andersmf@psykologi.uio.no.

DOI:10.1523/JNEUROSCI.3252-09.2009

Copyright $\odot 2009$ Society for Neuroscience $\quad$ 0270-6474/09/2915223-09\$15.00/0
}

often been limited by relatively small numbers of participants and use of either global measures (e.g., whole-brain volume) or only a restricted set of regions of interest (ROIs). Further, while atrophy has been demonstrated in mild cognitive impairment over 1 year or less (Schuff et al., 2009), it is not known whether brain changes driven solely by normal aging are large enough to be detectable over such short time intervals.

Longitudinal studies have reported annual gross brain volume decreases on the order of 0.2-0.5\% (Scahill et al., 2003; Ezekiel et al., 2004; Enzinger et al., 2005; Fotenos et al., 2005), while hippocampus, the most studied structure, shows annual atrophy rates from $0.79 \%$ to $2.0 \%$ (Jack et al., 1998; Scahill et al., 2003; Ezekiel et al., 2004; Raz et al., 2005; Du et al., 2006; Barnes et al., 2009). This tends to be higher than cross-sectional estimates (Scahill et al., 2003; Raz et al., 2005; Du et al., 2006). Entorhinal cortex longitudinal decline is $\sim 0.3-2.4 \%$ (Du et al., 2003, 2006; Ezekiel et al., 2004; Raz et al., 2005). Atrophy rates in other parts of the brain are seldom studied, with a few important exceptions (Pfefferbaum et al., 1998; Resnick et al., 2003; Raz et al., 2005; Driscoll et al., 2009). These studies describe atrophy in several regions, with prominent decline in prefrontal cortex, as well as decline in caudate nucleus, cerebellum, hippocampus, and association cortices (Raz et al., 2005) and parietal areas (Resnick et al., 2003), in addition to expansion of the ventricles (Pfefferbaum et al., 1998). In a recent study, significant atrophy was found in all 25 regional brain volumes tested, but over time periods extending as long as 10 years in some cases (Driscoll et al., 2009). An earlier study found significant ventricular enlargement over 1 year, but 
Table 1. Sample characteristics for the healthy elderly group

\begin{tabular}{llll}
\hline & Baseline & 1 year & 2 years \\
\hline$n$ & 142 & 142 & 89 \\
Sex (females/males) & $68 / 74$ & $68 / 74$ & $38 / 51$ \\
Age & $75.6(59.8-90.2)$ & $76.7(60.8-91.3)$ & $78.7(65.1-92.3)$ \\
MMSE & $29.2(25-30)$ & $29.1(24-30)$ & $29.1(26-30)$ \\
CDR sum of boxes & $0.03(0-0.5)$ & $0(0-0)$ & $0(0-0)$ \\
\hline
\end{tabular}

no detectable change in total or regional brain volumes (Resnick et al., 2000).

With newer analysis techniques, we may now possess the tools necessary to detect brain changes over short time intervals in healthy elderly. The aims of the present study were to determine whether brain changes in normal aging can be detected over 1 $(n=142)$ or $2(n=89)$ years, and if so, to determine which areas show the greatest changes, and whether rate of atrophy increases with age. A novel procedure for longitudinal change analysis was used, which enabled measures of change continuously across the cortex, as well as in 48 regions of interest. Atrophy in the healthy elderly was compared with 122 patients with mild to moderate Alzheimer's disease (AD).

\section{Materials and Methods}

The raw data used in the preparation of this article were obtained from the Alzheimer's Disease Neuroimaging Initiative (ADNI) database (www.loni.ucla.edu/ADNI). ADNI was launched in 2003 by the National Institute on Aging, the National Institute of Biomedical Imaging and Bioengineering, the Food and Drug Administration, private pharmaceutical companies, and nonprofit organizations. The primary goal of ADNI has been to test whether serial MRI, positron emission tomography, other biological markers, and clinical and neuropsychological assessment can be combined to measure the progression of mild cognitive impairment (MCI) and early AD. The Principal Investigator of this initiative is Michael W. Weiner, Veterans Affairs Medical Center and University of California-San Francisco. There are many coinvestigators, and subjects have been recruited from $>50$ sites across the U.S. and Canada. The initial goal of ADNI was to recruit 800 adults, including healthy elderly and MCI and AD patients, to participate and be followed for 2-3 years. For more information, see www.adni-info.org.

Sample. ADNI eligibility criteria are described at http://www.adni-info. org/index.php?option $=$ com_content\&task $=$ view\&id $=9 \&$ Itemid $=$ 43. Briefly, participants are 55-90 years of age, had an informant able to provide an independent evaluation of functioning, and spoke either English or Spanish. All subjects were willing and able to undergo test procedures including neuroimaging and agreed to longitudinal follow up. Specific psychoactive medications are excluded. General inclusion/ exclusion criteria are as follows for normal subjects: Mini-Mental State Examination (MMSE) (Folstein et al., 1975) scores between 24 and 30 (inclusive), Clinical Dementia Rating (CDR) (Morris, 1993) of 0, nondepressed, non-MCI, and nondemented. In addition, CDR sum of boxes (CDR-sb) was calculated as a measure of clinical functioning (does not need to be zero). To minimize the possibility of including individuals with early, preclinical $\mathrm{AD}$, only participants who had the same or better score on CDR-sb at the time of follow up were included. The subject pool was further restricted to those subjects for whom adequate processed and quality checked MR data were available by February 2009. Sample characteristics are presented in Table 1.

To be able to compare atrophy in the healthy elderly with $\mathrm{AD}$ patients, an $\mathrm{AD}$ group was included for selected analyses $[n=122,57 \mathrm{females} / 65$ males, at 1 year follow up: age $=76.5(56.0-90.0)$, MMSE $=20.7(8-29)$, $\mathrm{CDR}=1.0(0.5-3.0), \mathrm{CDR}-\mathrm{sb}=5.88(1-14)]$. These patients met the same eligibility criteria as the healthy elderly, had CDR scores of 0.5 or more, and met National Institute of Neurological and Communicative Disorders and Stroke/Alzheimer's Disease and Related Disorders Association criteria for probable $\mathrm{AD}$ (McKhann et al., 1984). As the mean age (76.5 vs 76.7 years, respectively) and the female/male ratio ( 0.88 vs 0.92 ) were almost identical between $\mathrm{AD}$ patients and healthy elderly, these variables were not regressed out in the analyses.

MR acquisition and analysis. All scans used for the present paper were from 1.5 T scanners. Data were collected across a variety of scanners with protocols individualized for each scanner, as defined at http://www.loni. ucla.edu/ADNI/Research/Cores/index.shtml. Raw DICOM MRI scans (including two T1-weighted volumes per case) were downloaded from the public ADNI site (http://www.loni.ucla.edu/ADNI/Data/index. shtml) and processed as described previously (Fennema-Notestine et al., 2009). Briefly, these data were reviewed for quality, automatically corrected for spatial distortion due to gradient nonlinearity (Jovicich et al., 2006) and $B_{1}$ field inhomogeneity (Sled et al., 1998), registered, and averaged to improve signal-to-noise ratio. Scans were segmented as described previously (Fischl et al., 2002, 2004) to yielding data for 11 different volumetric ROIs and four ventricular ROIs (right and left hemisphere regions measured separately), including the hippocampal formation [consisting of the dentate gyrus, CA fields, subiculum/parasubiculum, and the fimbria (Makris et al., 1999)]. The cortical surface was reconstructed using a semiautomated approach described previously (Dale and Sereno, 1993; Dale et al., 1999; Fischl et al., 1999a,b; Fischl and Dale, 2000; Salat et al., 2004), and parcellated as described by Fischl et al. (2004), labeling 33 cortical sulci and gyri (Desikan et al., 2006). The exact anatomical borders for the cortical ROIs can be found in Desikan et al. (2006), e.g., for the transverse temporal cortex (the rostral boundary was the rostral extent of the transverse temporal sulcus, the caudal boundary was the caudal portion of the insular cortex, and the lateral fissure and the superior temporal gyrus were used as the medial and lateral boundaries, respectively) and isthmus of the cingulate cortex [the rostral and caudal boundaries were the posterior division of the cingulate cortex and the parahippocampal gyrus, respectively, and the medial and lateral boundaries were the medial wall (area unknown) and the precuneus, respectively]. These surface-based cortical ROIs were thus generated by a different procedure from that used to estimate the other (volumetric) ROIs, which included the hippocampus, the cerebellum gray matter (GM), nine subcortical ROIs, and four ventricular ROIs.

For the analyses of the longitudinal volume changes, each participant's dual three-dimensional (3D) follow-up structural scans were rigid-body aligned, averaged, and affine aligned to the baseline scan. Nonlinear registration of the images was then performed, where voxel centers are moved about until a good match between the images is made. Several methods exist for effecting this, including fluid deformation (Miller et al., 1993; Christensen et al., 1996; Freeborough and Fox, 1998) and tensorbased morphometry (TBM) (Ashburner et al., 1999). For the results presented here, however, we applied a method (Holland et al., 2008) based on linear elasticity and closer in spirit to TBM. It proceeds essentially as follows. The images are heavily blurred (smoothed), making them almost identical, and a merit or potential function is calculated. This merit function expresses the intensity difference between the images at each voxel, and depends on the displacement field for the voxel centers of the image being transformed; it is also regularized to keep the displacement field spatially smooth. The merit function by design will have a minimum when the displacement field induces a good match between the images. The displacement field in general will turn cubic voxels into displaced irregular hexahedra whose volumes (Grandy, 1997) give the volume change field. The merit function is minimized efficiently using standard numerical methods. Having found a displacement field for the heavily blurred pair of images, the blurring is reduced and the procedure repeated, thus iteratively building up a better displacement field. Two important additions to this are: applying the final displacement field to the image being transformed, then nonlinearly registering the resultant image to the same target, and finally tracing back through the displacement fields thus calculated to find the net displacement field; and restricting to regions of interest and zooming when tissue structures are separated by only a voxel or two. These additional features enable very precise registration involving large or subtle deformations, even at small spatial scales with low boundary contrast. Although large deformations are allowed by multiple nonlinear registration (or relaxation) steps, nonphysical deformations are precluded because at each level of blurring the image undergoing deformation is constrained to conform to the target. 


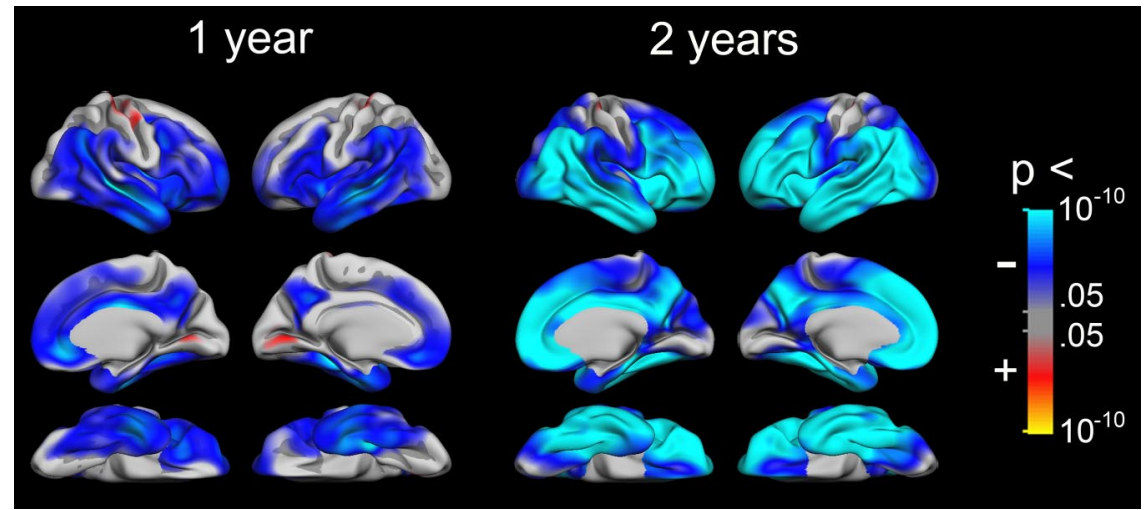

Figure 1. Cortical atrophy in healthy elderly. General linear models were used to test whether rates of atrophy for 1 and 2 years were significantly different from zero. The results were thresholded by a conventional criterion for correction for multiple comparisons (false discovery rate $<0.05$ ) and projected onto a semi-inflated template brain. Blue-cyan areas indicate cortical reduction, while red-yellow areas indicate expansion. As can be seen, significant volume reductions are found across large areas already after 1 year, and these were especially prominent in temporal and prefrontal areas.

Note that calculating the deformation field does not depend on initially segmenting tissue. This deformation field was used to align scans at the subvoxel level.

The follow-up aligned image underwent skull stripping and volumetric segmentations (subcortical structures, as well as hippocampus and cerebellum GM), with labels applied from the baseline scan. For the cortical reconstructions, surface coordinates for the white matter (WM) and pial boundaries were derived from the baseline images and mapped onto the follow-up images using the deformation field. Parcellations from the baseline image were then applied to the follow-up image. This resulted in a one-to-one correspondence between each vertex in the base image and the follow-up image. The procedure produces an estimate of the percentage cortical volume loss at each vertex and within each ROI. To the extent that regional cortical areas are relatively stable across time points, the volume change is likely driven almost exclusively by changes in thickness. The method has been validated in model studies of complex spherical-shell geometries with low contrast and noise, where a prescribed volume change is numerically estimated to accuracies of within $0.5 \%$ (D. Holland, unpublished work).

A challenge in cross-sectional MR studies of aging is that the contrast between GM and WM decreases with higher age (Salat et al., 2009). However, the longitudinal registration relies on matching intensities in serial images. A reduction in GM/WM contrast in elderly relative to younger participants should not impact the quality of the nonlinear registration, as long as the serial images have constant contrast. Change in cortical volume to first order arises from change in cortical thickness. To estimate it, the volume change field is sampled midway through the cortical layer. Conceivably, the primary source of error in elderly, relative to younger participants, would then be in the initial delineation of the cortical surface. However, it has recently been shown that the decrease in GM/WM contrast cannot explain the differences in cortical thickness between older and younger participants (Westlye et al., 2009).

Statistical analyses. Statistical analyses were performed by use of FreeSurfer (http://surfer.nmr.mgh.harvard.edu/) and SPSS 15.0. To reduce the number of comparisons, mean values of the left and right hemisphere were used for the ROI analyses. For the healthy elderly and the AD patients, change in brain morphometry was calculated as percentage change relative to baseline ( 1 and 2 years for the healthy elderly, 2 years for the $\mathrm{AD}$ patients). For the healthy elderly, statistical parametric continuous surface maps were computed by general linear models implemented in FreeSurfer and displayed on a semi-inflated template brain. The results were thresholded by a conventional criterion for correction for multiple comparisons (false discovery rate $<0.05$ ). For the healthy elderly and the AD patients, one-sample $t$ tests were used to test whether longitudinal atrophy rates within each ROI were different from zero. Independent-samples $t$ tests were used to compare rate of atrophy in healthy controls and AD patients. To visualize the areas that showed more than average and the areas that showed less than average atrophy as surface maps, mean cortical atrophy was calculated for each hemisphere in each group. This value was subtracted from the original surface maps, yielding a new map showing relative atrophy. To test whether the magnitude of atrophy in the temporal lobe versus the prefrontal cortex differed between the healthy elderly and the AD patients, ANOVA with 2 groups $\times 2$ regions were performed. Temporal lobe atrophy was calculated as mean rate of atrophy in inferior and middle temporal, entorhinal, parahippocampal, temporal pole, and fusiform cortices, as well as hippocampus and amygdala. Prefrontal atrophy was calculated as mean rate of atrophy in caudal and rostral anterior cingulate, caudal and rostral middle frontal, lateral, dorsal, and medial orbitofrontal, frontal pole, and superior frontal cortices, as well as pars opercularis, triangularis, and orbitalis (together forming the inferior frontal cortex) (see Fig. 4). Finally, Pearson correlations were performed between age and atrophy in each of the 48 ROIs in the healthy elderly.

\section{Results}

\section{Atrophy over 1 and 2 years: continuous surface analyses}

The statistical significance of 1 and 2 year volume changes in cortex across the brain surface is shown in Figure 1. As can be seen, large cortical areas decreased significantly over 1 year. These reductions were most prominent in the temporal lobe, the medial part of the superior frontal gyrus, and the orbitofrontal, precuneus, supramarginal, and inferior parietal cortices, as well as the inferior and part of the middle frontal cortex. Lateral parts of the superior frontal cortex, as well as precentral and postcentral gyri, superior parietal areas, occipital cortex, and lingual gyrus were largely spared. Over 2 years, the effects were stronger and spread to additional areas, involving all cortical areas except lingual cortex and precentral and postcentral gyri.

Percentage volume change in cortex over 1 and 2 years is shown in Figure 2. Annual atrophy rates varied across the cortex, but were typically $\sim 0.5 \%$ in affected areas, including most of the lateral and inferior parts of the temporal lobes, supramarginal gyrus, inferior parietal gyrus, precuneus, and inferior and middle frontal gyri, as well as the medial parts of the superior frontal gyrus. Atrophy over 2 years exceeded $1.0 \%$ in large areas.

For comparison purposes, rate of atrophy is presented for $\mathrm{AD}$ patients in Figure 2. Atrophy in the $\mathrm{AD}$ group was much larger than in the healthy elderly, with most areas showing $1 \%$ annual change or more. This was especially evident in the lateral temporal cortex and an area around posterior cingulate/inferior precuneus/retrosplenial cortex ( $>2.5 \%$ annual change). Less atrophy was seen around the central sulcus, pericalcarine sulcus, and lingual gyrus. To compare the pattern of atrophy between the healthy controls and the AD patients, mean cortical atrophy was calculated for each hemisphere in each group. This value was subtracted from the surface maps, yielding a new map highlighting areas that show greater and lesser rates of atrophy than average for that group. These maps illustrate the parts of the cortex that show relatively higher and relatively lower rates of atrophy within each group (Fig. 3). Within the AD group, higher than average atrophy was seen in the inferior and middle temporal gyri. Within the healthy elderly group, higher than average atrophy was observed in the same areas, but also in the temporal pole, medial orbitofrontal, rostral middle frontal, and supramarginal 


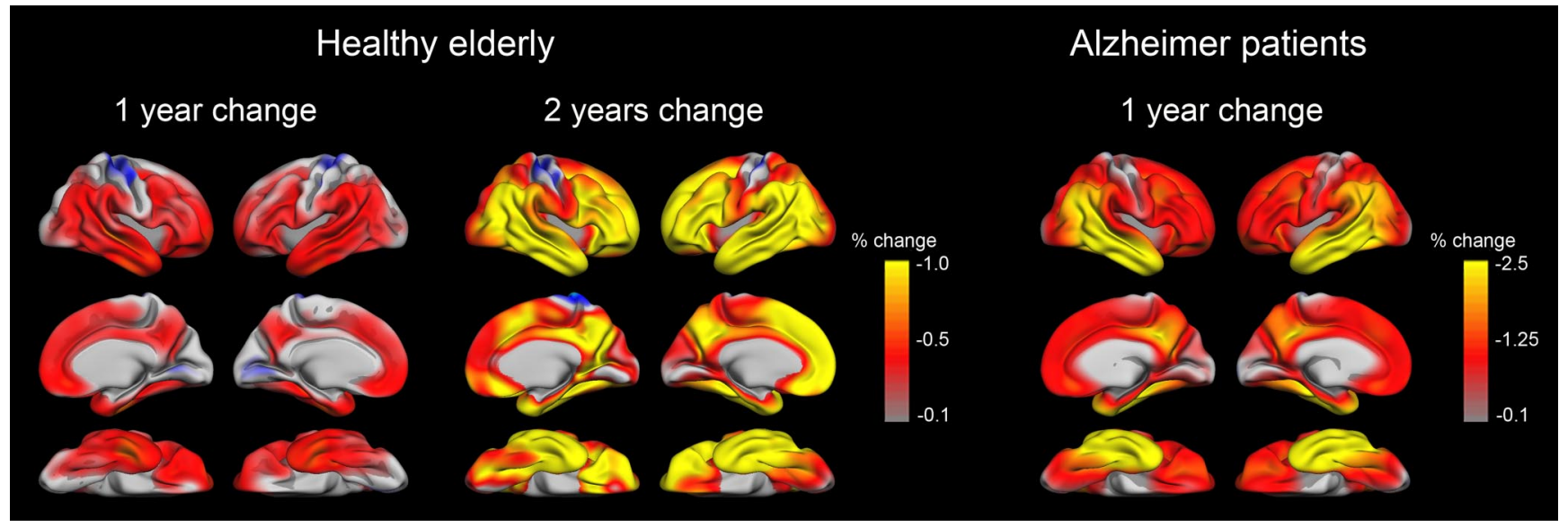

Figure 2. Rate of change in healthy elderly and AD patients. The left panel shows annual longitudinal atrophy in healthy elderly calculated as percentage volume change relative to baseline, while the middle panel shows longitudinal atrophy over 2 years. Annual change of $\sim 0.5 \%$ can be seen across large sections of the brain surface, e.g., in temporal and prefrontal areas. The right panel shows 1 year atrophy in AD patients. The areas of change in the healthy elderly are overlapping with areas most strongly affected in Alzheimer's disease, e.g., temporal lobe cortex. However, areas that are not especially affected in AD, e.g., prefrontal cortex, also undergo large changes in healthy aging. Please note that to allow optimal visualization of regional variation, the color scale is different for the healthy elderly and the AD groups.

cortices, as well as parts of the medial superior frontal gyrus. Less than average atrophy was seen in cuneus, pericalcarine sulcus, and lingual gyrus, as well as the central sulcus. Results of formal statistical tests of differences in rates of atrophy between healthy controls and $\mathrm{AD}$ patients are described below.

\section{Atrophy over 1 and 2 years:}

\section{ROI analyses}

Percentage annual volume change in each of the 48 ROIs is shown in Table 2 for healthy elderly. Median atrophy for 1 year was $-0.38 \%$, not including the ventricular ROIs. Hippocampus $(-0.84 \%)$ and amygdala $(-0.81 \%)$ showed the largest reductions, followed by thalamus $(-0.69 \%)$. Cerebellum gray matter $(-0.35 \%)$, the brainstem $(-0.31 \%)$, and caudate nucleus $(-0.24 \%)$ showed the least 1 year change of the volumetric ROIs. The inferior lateral (5.47\%), lateral (4.40\%), and third (3.07\%) ventricles showed the largest changes overall, with no significant change in the fourth $(0.71 \%)$ ventricle. Of the cortical ROIs, the frontal pole $(-0.59 \%)$, temporal pole $(-0.56 \%)$, banks of the superior temporal sulcus $(-0.55 \%)$, and entorhinal cortex $(-0.55 \%)$ changed the most, while postcentral, pericalcarine, lingual, cuneus, and precentral were well preserved $(\leq 0.1 \%)$.

For comparison purposes, 1 year volume change in each ROI was calculated for the $\mathrm{AD}$ group also (Table 3 ). All ROIs except the pericalcarine sulcus showed significant change. Median atrophy for 1 year was $-1.20 \%$, not including the ventricular ROIs. Amygdala $(-3.79 \%)$ and hippocampus $(-3.75 \%)$ showed the largest reductions, followed by inferior and middle temporal gyri $(-2.97$ and $-2.87 \%$, respectively). Entorhinal and parahippocampal cortices also showed well above average atrophy $(-2.42$ and $-2.05 \%$, respectively). Pericalcarine $(-0.16 \%)$, postcentral $(-0.27 \%)$, and cuneus $(-0.5 \%)$ were the three ROIs showing the least atrophy, but of these only pericalcarine did not change at $p<0.01(p=0.030)$. The inferior lateral $(16.26 \%)$, lateral (10.35\%), and third (6.82\%) ventricles showed the largest changes overall.

Differences in rates of atrophy were formally tested between healthy elderly and $\mathrm{AD}$ patients. $\mathrm{AD}$ patients had significantly $(p<$ 0.01 ) higher rates of change than the healthy elderly for all ROIs except fourth ventricle (supplemental Table 1, available at www. jneurosci.org as supplemental material). In terms of $t$ scores, inferior temporal $(t=13.25)$, fusiform $(t=13.15)$, middle temporal $(t=$ 12.81), parahippocampal $(t=12.69)$, and entorhinal $(t=11.93)$ showed the largest effects of the cortical ROIs, while hippocampus $(t=12.80)$, the inferior lateral ventricles $(t=9.74)$, and amygdala $(t=8.32)$ showed the largest effects for the volumetric ROIs.

Interaction between the groups and temporal versus frontal atrophy rates were tested by ANOVA with two groups (healthy elderly and $\mathrm{AD}) \times$ two brain regions (frontal and temporal). A highly significant interaction was found $\left(F_{(1,256)}=119.78, p<\right.$ $10^{-22}$ ), caused by a higher rate of temporal than frontal atrophy in the $\mathrm{AD}$ group and a comparable rate of temporal versus frontal atrophy rate in the healthy elderly (Fig. 4). 
Table 2. Percentage volume change over 1 and 2 years in healthy elderly

\begin{tabular}{|c|c|c|c|c|}
\hline & \multicolumn{2}{|l|}{1 year } & \multicolumn{2}{|l|}{2 years } \\
\hline & $\begin{array}{l}\text { Longitudinal } \\
\text { change (\%) }\end{array}$ & $p$ & $\begin{array}{l}\text { Longitudinal } \\
\text { change (\%) }\end{array}$ & $p$ \\
\hline \multicolumn{5}{|l|}{ Volumetric ROIs } \\
\hline Accumbens & -0.57 & $<10^{-8}$ & -1.40 & $<10^{-19}$ \\
\hline Amygdala & -0.81 & $<10^{-9}$ & -2.02 & $<10^{-13}$ \\
\hline Brainstem & -0.31 & $<10^{-5}$ & -0.86 & $<10^{-12}$ \\
\hline Caudate & -0.24 & $>0.01^{\text {n.s. }}$ & -0.62 & $<0.005$ \\
\hline Cerebellum GM & -0.35 & $<10^{-8}$ & -0.94 & $<10^{-15}$ \\
\hline Cerebellum WM & -0.57 & $<10^{-14}$ & -1.22 & $<10^{-20}$ \\
\hline Cerebral WM & -0.58 & $<10^{-16}$ & -1.15 & $<10^{-19}$ \\
\hline Hippocampus & -0.84 & $<10^{-14}$ & -1.76 & $<10^{-15}$ \\
\hline Pallidum & -0.40 & $<10^{-11}$ & -0.75 & $<10^{-13}$ \\
\hline Putamen & -0.43 & $<10^{-8}$ & -0.92 & $<10^{-16}$ \\
\hline Thalamus & -0.69 & $<10^{-16}$ & -1.42 & $<10^{-21}$ \\
\hline \multicolumn{5}{|l|}{ Ventricular ROls } \\
\hline Lat vent & 4.40 & $<10^{-21}$ & 9.42 & $<10^{-19}$ \\
\hline Inf lat vent & 5.47 & $<10^{-15}$ & 12.11 & $<10^{-18}$ \\
\hline 3rd ventricle & 3.07 & $<10^{-12}$ & 6.60 & $<10^{-14}$ \\
\hline 4th ventricle & 0.71 & $>0.01^{\text {n.s. }}$ & 2.18 & $<10^{-4}$ \\
\hline \multicolumn{5}{|l|}{ Surface ROls } \\
\hline Cingulate, caudal ant & -0.27 & $<0.001$ & -0.91 & $<10^{-12}$ \\
\hline Cingulate, rostral ant & -0.34 & $<10^{-4}$ & -0.92 & $<10^{-12}$ \\
\hline Cingulate, posterior & -0.25 & $<0.001$ & -0.79 & $<10^{-7}$ \\
\hline Cingulate, isthmus & -0.20 & $<0.005$ & -0.63 & $<10^{-7}$ \\
\hline Frontal, superior & -0.31 & $<0.005$ & -0.93 & $<10^{-11}$ \\
\hline Frontal, caudal middle & -0.40 & $<10^{-6}$ & -0.99 & $<10^{-12}$ \\
\hline Frontal, rostral middle & -0.32 & $<10^{-4}$ & -0.98 & $<10^{-13}$ \\
\hline Frontal, pars opercularis & -0.38 & $<10^{-7}$ & -1.04 & $<10^{-14}$ \\
\hline Frontal, pars triangularis & -0.37 & $<10^{-6}$ & -1.08 & $<10^{-15}$ \\
\hline Frontal, pars orbitalis & -0.38 & $<10^{-4}$ & -1.11 & $<10^{-13}$ \\
\hline Frontal, lateral orbital & -0.42 & $<10^{-6}$ & -1.07 & $<10^{-16}$ \\
\hline Frontal, medial orbital & -0.39 & $<10^{-5}$ & -0.98 & $<10^{-14}$ \\
\hline Frontal, pole & -0.59 & $<10^{-5}$ & -1.30 & $<10^{-11}$ \\
\hline Parietal, precentral gyrus & -0.10 & $>0.01^{\text {n.s. }}$ & -0.48 & $<10^{-4}$ \\
\hline Parietal, postcentral gyrus & 0.09 & $>0.01^{\text {n.s. }}$ & 0.01 & $>0.01^{\text {n.s. }}$ \\
\hline Parietal, paracentral gyrus & -0.17 & $>0.01^{\text {n.s. }}$ & -0.52 & $<0.001$ \\
\hline Parietal, superior & -0.18 & $>0.01^{\text {n.s. }}$ & -0.68 & $<10^{-5}$ \\
\hline Parietal, inferior & -0.40 & $<10^{-7}$ & -1.03 & $<10^{-13}$ \\
\hline Parietal, supramarginal & -0.40 & $<10^{-6}$ & -0.98 & $<10^{-10}$ \\
\hline Parietal, precuneus & -0.30 & $<10^{-6}$ & -0.82 & $<10^{-10}$ \\
\hline Temporal, parahippocampal & -0.36 & $<10^{-6}$ & -1.08 & $<10^{-18}$ \\
\hline Temporal, entorhinal & -0.55 & $<10^{-7}$ & -1.20 & $<10^{-11}$ \\
\hline Temporal, pole & -0.56 & $<10^{-5}$ & -1.17 & $<10^{-9}$ \\
\hline Temporal, superior & -0.43 & $<10^{-7}$ & -1.00 & $<10^{-13}$ \\
\hline Temporal, middle & -0.47 & $<10^{-8}$ & -1.25 & $<10^{-16}$ \\
\hline Temporal, inferior & -0.47 & $<10^{-9}$ & -1.18 & $<10^{-17}$ \\
\hline Temporal, transverse & -0.31 & $<0.005$ & -0.79 & $<10^{-8}$ \\
\hline Temporal, banks sup temp sulc & -0.55 & $<10^{-11}$ & -1.32 & $<10^{-15}$ \\
\hline Temporal, fusiform & -0.38 & $<10^{-9}$ & -0.98 & $<10^{-18}$ \\
\hline Occipital, lateral & -0.19 & $<0.005$ & -0.66 & $<10^{-9}$ \\
\hline Occipital, pericalcarine & 0.11 & $>0.01^{\text {n.s. }}$ & -0.18 & $>0.01^{\text {n.s. }}$ \\
\hline Occipital, lingual & -0.02 & $>0.01^{\text {n.s. }}$ & -0.35 & $<10^{-4}$ \\
\hline Occipital, cuneus & -0.04 & $>0.01^{\text {n.s. }}$ & -0.40 & $<10^{-4}$ \\
\hline
\end{tabular}

n.s., Not significant. Ant, Anterior; inf, inferior; lat, lateral; sulc, sulcus; sup, superior; temp, temporal; vent, ventricle.

\section{Effects of age on regional atrophy rates}

Correlation analyses between age and 1 year atrophy in each of the ROIs were performed. The results are presented in Table 4 and scatter plots for selected structures in Figure 5. We focus here on the 1 year changes, since the statistical power was lower for 2 years. Significant correlations between age and rates of atrophy were found for 32 ROIs. For the volumetric structures, hippocampus, cerebellum gray and white matter, cerebral white matter, thalamus, and pallidum atrophy were related to age, as was expansion of the inferior lateral ventricles. Of the cortical structures, regions demonstrating significant relationships between age and rate of atrophy included entorhinal, transverse
Table 3. Percentage volume change over 1 year in the AD group

\begin{tabular}{|c|c|c|}
\hline & Longitudinal change (\%) & $p$ \\
\hline \multicolumn{3}{|l|}{ Volumetric ROls } \\
\hline Accumbens & -2.02 & $<10^{-19}$ \\
\hline Amygdala & -3.79 & $<10^{-19}$ \\
\hline Brainstem & -0.65 & $<10^{-16}$ \\
\hline Caudate & -1.49 & $<10^{-13}$ \\
\hline Cerebellum GM & -0.99 & $<10^{-25}$ \\
\hline Cerebellum WM & -0.95 & $<10^{-18}$ \\
\hline Cerebral WM & -1.42 & $<10^{-30}$ \\
\hline Hippocampus & -3.75 & $<10^{-35}$ \\
\hline Pallidum & -0.90 & $<10^{-15}$ \\
\hline Putamen & -1.45 & $<10^{-22}$ \\
\hline Thalamus & -1.51 & $<10^{-28}$ \\
\hline \multicolumn{3}{|l|}{ Ventricular ROls } \\
\hline Lat vent & 10.35 & $<10^{-28}$ \\
\hline Inf lat vent & 16.26 & $<10^{-33}$ \\
\hline 3rd ventricle & 6.82 & $<10^{-21}$ \\
\hline 4th ventricle & 1.61 & $<0.001$ \\
\hline \multicolumn{3}{|l|}{ Surface ROls } \\
\hline Cingulate, caudal ant & -1.14 & $<10^{-17}$ \\
\hline Cingulate, rostral ant & -1.20 & $<10^{-18}$ \\
\hline Cingulate, posterior & -1.57 & $<10^{-21}$ \\
\hline Cingulate, isthmus & -1.54 & $<10^{-23}$ \\
\hline Frontal, superior & -1.20 & $<10^{-15}$ \\
\hline Frontal, caudal middle & -1.60 & $<10^{-20}$ \\
\hline Frontal, rostral middle & -1.23 & $<10^{-15}$ \\
\hline Frontal, pars opercularis & -1.31 & $<10^{-18}$ \\
\hline Frontal, pars triangularis & -1.10 & $<10^{-17}$ \\
\hline Frontal, pars orbitalis & -1.06 & $<10^{-<1}$ \\
\hline Frontal, lateral orbital & -1.47 & $<10^{-18}$ \\
\hline Frontal, medial orbital & -1.19 & $<10^{-17}$ \\
\hline Frontal, pole & -1.38 & $<10^{-10}$ \\
\hline Parietal, precentral gyrus & -0.69 & $<10^{-10}$ \\
\hline Parietal, postcentral gyrus & -0.27 & 0.01 \\
\hline Parietal, paracentral gyrus & -0.76 & $<10^{-11}$ \\
\hline Parietal, superior & -1.26 & $<10^{-18}$ \\
\hline Parietal, inferior & -2.10 & $<10^{-27}$ \\
\hline Parietal, supramarginal & -1.74 & $<10^{-21}$ \\
\hline Parietal, precuneus & -1.61 & $<10^{-24}$ \\
\hline Temporal, parahippocampal & -2.05 & $<10^{-34}$ \\
\hline Temporal, entorhinal & -2.42 & $<10^{-36}$ \\
\hline Temporal, pole & -2.54 & $<10^{-26}$ \\
\hline Temporal, superior & -1.63 & $<10^{-23}$ \\
\hline Temporal, middle & -2.87 & $<10^{-31}$ \\
\hline Temporal, inferior & -2.97 & $<10^{-32}$ \\
\hline Temporal, transverse & -0.59 & $<10^{-6}$ \\
\hline Temporal, banks sup temp sulc & -2.53 & $<10^{-28}$ \\
\hline Temporal, fusiform & -2.15 & $<10^{-33}$ \\
\hline Occipital, lateral & -0.96 & $<10^{-19}$ \\
\hline Occipital, pericalcarine & -0.16 & $>0.01^{\mathrm{n} . \mathrm{s}}$ \\
\hline Occipital, lingual & -0.67 & $<10^{-15}$ \\
\hline Occipital, cuneus & -0.50 & $<10^{-9}$ \\
\hline
\end{tabular}

temporal, pars opercularis, isthmus cingulate, middle temporal, pars triangularis, parahippocampal, superior temporal, posterior cingulate, caudal middle frontal, precentral, fusiform, inferior parietal, temporal pole, precuneus, supramarginal, lateral orbitofrontal, superior parietal, postcentral, caudal anterior cingulate, medial orbitofrontal, pericalcarine, lateral occipital, and inferior temporal cortices, as well as the banks of the superior temporal sulcus.

\section{Discussion}

Volumetric reductions of the cerebral cortex and subcortical brain structures, as well as expansion of the ventricles, were seen 

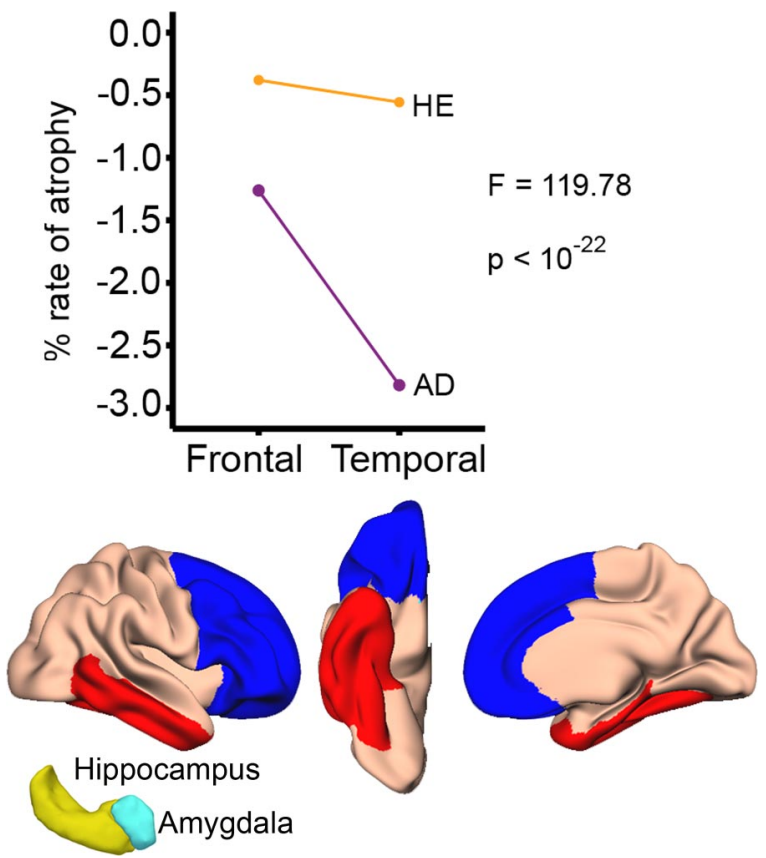

Figure 4. Frontal versus temporal effects of healthy aging and Alzheimer's disease. The line graphs show rate of atrophy for the frontal and the temporal regions in healthy elderly and AD patients. The regions are shown below (blue: frontal, red: temporal). In addition to the cortical areas shown, hippocampus and amygdala were included in the temporal region (yellow and cyan 3D figures). ANOVA showed a significant interaction between group and region: While the $\mathrm{AD}$ patients showed more atrophy in the temporal $(-2.82 \%, \mathrm{SE}=0.14)$ than the frontal regions $(-1.26 \%, \mathrm{SE}=0.11)$, the rate of atrophy between these two regions was comparable in the healthy controls (temporal: $-0.56 \%, \mathrm{SE}=0.07 /$ frontal: $-0.38 \%, \mathrm{SE}=0.07$ ).

in healthy aging after only 1 year. While this has been shown previously for MCI and AD patients (McDonald et al., 2009; Schuff et al., 2009), the present report is the first to demonstrate detectable atrophy in a range of brain areas over only 1 year in healthy participants. Some of the changes seen, e.g., in the temporal lobe, may be due to preclinical $\mathrm{AD}$, while others, e.g., in the prefrontal cortex, likely reflects the nonpathological aging process. This is discussed below.

The present atrophy rates for hippocampus $(0.84 \%)$ and entorhinal cortex $(0.55 \%)$ are comparable to previous studies (Jack et al., 1998; Du et al., 2003, 2006; Scahill et al., 2003; Ezekiel et al., 2004; Raz et al., 2005; Barnes et al., 2009), supporting the feasibility of pooling data across multiple sites resulting in sensitive and realistic atrophy estimates. Generally, volumetric ROIs showed more decline than surface-based cortical ROIs, with the hippocampus showing the greatest 1 year reduction. Hippocampal volume has been found to correlate with age in cross-sectional (Good et al., 2001; Raz et al., 2004; Walhovd et al., 2005) and longitudinal (Jack et al., 1998; Resnick et al., 2003; Scahill et al., 2003; Ezekiel et al., 2004; Raz et al., 2005; Du et al., 2006; Driscoll et al., 2009) studies, but usually not more strongly than the other volumetric structures measured (Raz et al., 2005). However, in a recent cross-sectional multisample study, estimated percentage decline per decade in the latter half of the lifespan was larger for hippocampus than any other volumetric structure (Walhovd et al., 2009). In the present study, amygdala, thalamus, putamen, and pallidum also showed longitudinal decline. Several crosssectional studies have shown substantial reductions in the volumes of thalamus, amygdala, and putamen (Greenberg et al., 2008; Gunning-Dixon et al., 1998; Raz et al., 2003; Sullivan et al., 2004; Van Der Werf et al., 2001; Walhovd et al., 2005, 2009), as
Table 4. Pearson correlations between age and 1 year volume change in healthy elderly

\begin{tabular}{|c|c|}
\hline & 1 year change \\
\hline \multicolumn{2}{|l|}{ Volumetric ROls } \\
\hline Accumbens & -0.13 \\
\hline Amygdala & -0.16 \\
\hline Brainstem & -0.15 \\
\hline Caudate & -0.06 \\
\hline Cerebellum GM & -0.20 \\
\hline Cerebellum WM & -0.17 \\
\hline Cerebral WM & -0.17 \\
\hline Hippocampus & -0.33 \\
\hline Pallidum & -0.17 \\
\hline Putamen & -0.14 \\
\hline Thalamus & -0.18 \\
\hline \multicolumn{2}{|l|}{ Ventricular ROls } \\
\hline Lateral ventricles & 0.08 \\
\hline Inf lateral ventricles & 0.21 \\
\hline 3rd ventricle & 0.03 \\
\hline 4th ventricle & 0.08 \\
\hline \multicolumn{2}{|l|}{ Surface ROls } \\
\hline Cingulate, caudal ant & -0.19 \\
\hline Cingulate, rostral ant & -0.11 \\
\hline Cingulate, posterior & -0.22 \\
\hline Cingulate, isthmus & -0.25 \\
\hline Frontal, superior & -0.13 \\
\hline Frontal, caudal middle & -0.21 \\
\hline Frontal, rostral middle & -0.13 \\
\hline Frontal, pars opercularis & -0.26 \\
\hline Frontal, pars triangularis & -0.23 \\
\hline Frontal, pars orbitalis & -0.08 \\
\hline Frontal, lateral orbital & -0.19 \\
\hline Frontal, medial orbital & -0.18 \\
\hline Frontal, pole & -0.11 \\
\hline Parietal, precentral gyrus & -0.21 \\
\hline Parietal, postcentral gyrus & -0.19 \\
\hline Parietal, paracentral gyrus & -0.13 \\
\hline Parietal, superior & -0.19 \\
\hline Parietal, inferior & -0.20 \\
\hline Parietal, supramarginal & -0.19 \\
\hline Parietal, precuneus & -0.20 \\
\hline Temporal, parahippocampal & -0.23 \\
\hline Temporal, entorhinal & -0.32 \\
\hline Temporal, pole & -0.20 \\
\hline Temporal, superior & -0.22 \\
\hline Temporal, middle & -0.23 \\
\hline Temporal, inferior & -0.17 \\
\hline Temporal, transverse & -0.30 \\
\hline Temporal, banks sup temp sulc & -0.23 \\
\hline Temporal, fusiform & -0.20 \\
\hline Occipital, lateral & -0.17 \\
\hline Occipital, pericalcarine & -0.18 \\
\hline Occipital, lingual & -0.16 \\
\hline Occipital, cuneus & -0.13 \\
\hline
\end{tabular}

$\overline{\text { Negative correlations between age and rate of atrophy, and positive correlations between age and rate of ventricular }}$ expansion are generally found, showing that increasing age is associated with more atrophy and increased ventricular size. Italic indicates $p<0.05(|r| \geq 0.17)$. Bold indicates $p<0.01(|r| \geq 0.21)$. Bold italic indicates $p<0.001$ $(|r| \geq 0.27)$. Ant, Anterior; inf, inferior; sulc, sulcus; sup, superior; temp, temporal.

well as in pallidum after 50 years of age (Walhovd et al., 2005, 2009), but discrepant findings regarding thalamus and pallidum have been reported (Jernigan et al., 1991; Gunning-Dixon et al., 1998; Luft et al., 1999; Jernigan et al., 2001; Raz et al., 2003).

Hippocampus and amygdala were the two volumetric structures that showed the largest annual decline in the present study, and these are known to show accelerated atrophy early in $\mathrm{AD}$ (de Leon et al., 1989; Jack et al., 1999; Fischl et al., 2002; Barnes et al., 

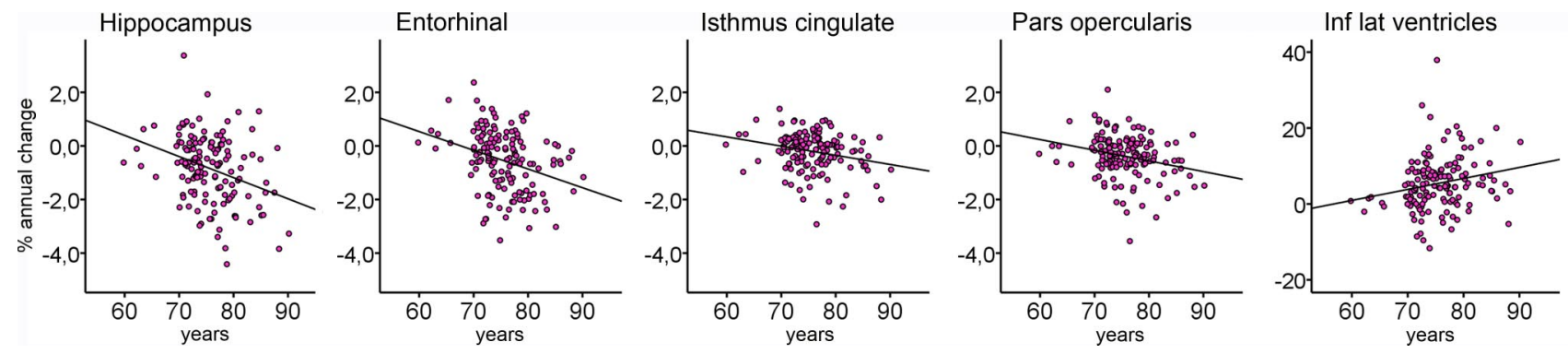

Figure 5. Relationships between age and rate of atrophy in healthy elderly. Scatter plots for selected ROls showing the relationship between age and rates of annual volumetric reductions.

2009; Fennema-Notestine et al., 2009; McDonald et al., 2009). A recent meta-analysis estimated annual hippocampal reductions to be $4.66 \%$ in $\mathrm{AD}$ (Barnes et al., 2009). These were also the ROIs showing the largest volume decrease in the $\mathrm{AD}$ patients in the present study, with annual rates of decline of $3.75 \%$ for hippocampus and $3.79 \%$ for amygdala in the AD sample. Of the surface-based cortical ROIs showing substantial decline, entorhinal, parahippocampal, and lateral temporal, as well as inferior parietal, supramarginal, and fusiform areas, are all found to be vulnerable in early stages of AD (Singh et al., 2006; Du et al., 2007; Fennema-Notestine et al., 2009; McEvoy et al., 2009; McDonald et al., 2009). An important question regards the extent to which atrophy in these areas in healthy elderly is an indication of preclinical $\mathrm{AD}$. We cannot rule out with certainty that degenerative processes associated with early $\mathrm{AD}$ caused some of the observed atrophy. However, reductions were also seen in areas relatively less affected by early AD. For instance, large effects were found in inferior, middle, and superior frontal cortices. Previous studies of the ADNI sample did not find differences in thickness between single domain amnestic MCI and controls in these areas (Fennema-Notestine et al., 2009), nor did these areas show longitudinal increases in atrophy in MCI participants showing mild functional impairment (CDR-sb of 1 or less) (McDonald et al., 2009). Still, a recent study found prefrontal cortical volume to predict dementia (Burgmans et al., 2009a), and AD involves frontal atrophy (Singh et al., 2006; Du et al., 2007; Fjell et al., 2008; Dickerson et al., 2009). An early longitudinal study found that while temporal atrophy was seen in presymptomatic and mildly affected patients, frontal involvement occurred later in the disease (Scahill et al., 2002). Another study found demented patients to show annual atrophy rates exceeding $0.4 \%$ in large areas of the brain, including the frontal cortex (Buckner et al., 2005). In the present study, more atrophy was observed in $\mathrm{AD}$ than in healthy controls in almost all ROIs, including prefrontal cortex. However, temporal lobe atrophy was the most prominent in $\mathrm{AD}$, while healthy aging was characterized by comparable rates of atrophy in the temporal lobe and the frontal cortex. The temporal changes seen in the healthy elderly group may be related to instances of preclinical $\mathrm{AD}$, while the frontal changes are more prominent than what would be expected due to AD. Significant decline was also observed in thalamus, accumbens, and cerebral and cerebellar white matter, areas that are not among the most vulnerable in early phases of AD (Fennema-Notestine et al., 2009). In the AD patients included in the present study, cerebellum WM was the 39 th most vulnerable region, while thalamus was the 22 nd and accumbens the 14th. Thus, it is unlikely that all the brain changes found here in the healthy elderly group can be attributed to preclinical AD. We believe that many of the changes observed in healthy aging are caused by processes unrelated to degenerative disease. Consistent with this, a recent study reported that the transition from healthy elderly to MCI was associated with a unique pattern of longitudinal change, including increased ventricular size and reductions in temporal gray matter, orbitofrontal areas, and temporal association cortices, including the hippocampus (Driscoll et al., 2009). The pattern of atrophy over several years in healthy elderly not developing MCI was widespread but did not resemble the pattern associated with MCI (Driscoll et al., 2009). As further follow-up data become available, the hypothesis that the temporal changes seen in the elderly are related to preclinical AD, while the frontal changes are less associated with incipient disease, can be tested. Since it is possible that structural brain changes due to degenerative disease may be manifested long before cognitive symptoms are detectable, follow-up cognitive and clinical data for several years will be invaluable (Burgmans et al., 2009b).

\section{Accelerated atrophy with higher age}

Older age was related to increased atrophy and more ventricular expansion. This is consistent with cross-sectional reports of nonlinear volumetric changes (Allen et al., 2005; Jernigan and Gamst, 2005; Walhovd et al., 2005, 2009; Fjell et al., 2009b). However, cross-sectional studies of cortical thickness have mostly reported linear age changes over the adult lifespan (Salat et al., 2004; Fjell et al., 2009a). It is possible that longitudinal designs are more sensitive to accelerated decline in the oldest old, especially if the effects are of moderate strength and evident only in the latest part of the lifespan. The correlations between age and rate of change seldom exceeded \pm 0.30 . Interestingly, the strongest correlations between age and rate of atrophy were found in entorhinal cortex and the hippocampus, and significant correlations were also found in isthmus of the cingulate, middle temporal, and parahippocampal gyri, all of which are affected in early stages of AD. Since the occurrence of preclinical $\mathrm{AD}$ is much higher among 70 and 80 -year-olds than among 50- and 60-year-olds, it is possible that the pattern of accelerated longitudinal atrophy in aging is related to preclinical $\mathrm{AD}$ in a subgroup of the participants, even though cognitive symptoms are not yet manifested. This interpretation is in agreement with another recent study where elderly cognitively normal participants showed a pattern of atrophy more similar to that seen in $\mathrm{AD}$ than did younger participants, i.e., more temporal lobe atrophy (Davatzikos et al., 2009).

\section{Conclusion}

The present results show that widespread changes in brain structure can be found in healthy elderly over only 1 year, and that atrophy accelerates with advanced age. Some of the atrophy occurred in areas vulnerable to $\mathrm{AD}$ and some in areas less characteristic of the disease in early stages. This suggests that the changes are not primarily driven by degenerative processes associated with $\mathrm{AD}$, although it is likely that preclinical changes associated 
with $\mathrm{AD}$ are superposed on changes due to normal aging in some subjects. Indeed, the pattern of accelerated atrophy with increasing age is probably influenced by occurrence of preclinical $\mathrm{AD}$ in a subgroup of the most elderly participants, and this may especially be reflected in the temporal lobe. Nevertheless, this study shows that decreases in brain volume in healthy aging can be observed in almost all brain areas over 1-2 years.

\section{References}

Allen JS, Bruss J, Brown CK, Damasio H (2005) Normal neuroanatomical variation due to age: the major lobes and a parcellation of the temporal region. Neurobiol Aging 26:1245-1260; discussion 1279-1282.

Ashburner J, Andersson JL, Friston KJ (1999) High-dimensional image registration using symmetric priors. Neuroimage 9:619-628.

Barnes J, Bartlett JW, van de Pol LA, Loy CT, Scahill RI, Frost C, Thompson P, Fox NC (2009) A meta-analysis of hippocampal atrophy rates in Alzheimer's disease. Neurobiol Aging 30:1711-1723.

Buckner RL, Snyder AZ, Shannon BJ, LaRossa G, Sachs R, Fotenos AF, Sheline YI, Klunk WE, Mathis CA, Morris JC, Mintun MA (2005) Molecular, structural, and functional characterization of Alzheimer's disease: evidence for a relationship between default activity, amyloid, and memory. J Neurosci 25:7709-7717.

Burgmans S, van Boxtel MP, Smeets F, Vuurman EF, Gronenschild EH, Verhey FR, Uylings HB, Jolles J (2009a) Prefrontal cortex atrophy predicts dementia over a six-year period. Neurobiol Aging 30:1413-1419.

Burgmans S, van Boxtel MP, Vuurman EF, Smeets F, Gronenschild EH, Uylings HB, Jolles J (2009b) The prevalence of cortical gray matter atrophy may be overestimated in the healthy aging brain. Neuropsychology 23:541-550.

Christensen GE, Rabbitt RD, Miller MI (1996) Deformable templates using large deformation kinematics. IEEE Trans Image Process 5:1435-1447.

Dale AM, Sereno MI (1993) Improved localization of cortical activity by combining EEG and MEG with MRI cortical surface reconstruction: a linear approach. J Cogn Neurosci 5:162-176.

Dale AM, Fischl B, Sereno MI (1999) Cortical surface-based analysis. I. Segmentation and surface reconstruction. Neuroimage 9:179-194.

Davatzikos C, Xu F, An Y, Fan Y, Resnick SM (2009) Longitudinal progression of Alzheimer's-like patterns of atrophy in normal older adults: the SPARE-AD index. Brain 132:2026-2035.

de Leon MJ, George AE, Stylopoulos LA, Smith G, Miller DC (1989) Early marker for Alzheimer's disease: the atrophic hippocampus. Lancet 2:672-673.

Desikan RS, Ségonne F, Fischl B, Quinn BT, Dickerson BC, Blacker D, Buckner RL, Dale AM, Maguire RP, Hyman BT, Albert MS, Killiany RJ (2006) An automated labeling system for subdividing the human cerebral cortex on MRI scans into gyral based regions of interest. Neuroimage 31:968-980.

Dickerson BC, Bakkour A, Salat DH, Feczko E, Pacheco J, Greve DN, Grodstein F, Wright CI, Blacker D, Rosas HD, Sperling RA, Atri A, Growdon JH, Hyman BT, Morris JC, Fischl B, Buckner RL (2009) The cortical signature of Alzheimer's disease: regionally specific cortical thinning relates to symptom severity in very mild to mild $\mathrm{AD}$ dementia and is detectable in asymptomatic amyloid-positive individuals. Cereb Cortex 19:497-510.

Driscoll I, Davatzikos C, An Y, Wu X, Shen D, Kraut M, Resnick SM (2009) Longitudinal pattern of regional brain volume change differentiates normal aging from MCI. Neurology 72:1906-1913.

Du AT, Schuff N, Zhu XP, Jagust WJ, Miller BL, Reed BR, Kramer JH, Mungas D, Yaffe K, Chui HC, Weiner MW (2003) Atrophy rates of entorhinal cortex in AD and normal aging. Neurology 60:481-486.

Du AT, Schuff N, Chao LL, Kornak J, Jagust WJ, Kramer JH, Reed BR, Miller BL, Norman D, Chui HC, Weiner MW (2006) Age effects on atrophy rates of entorhinal cortex and hippocampus. Neurobiol Aging 27:733-740.

Du AT, Schuff N, Kramer JH, Rosen HJ, Gorno-Tempini ML, Rankin K, Miller BL, Weiner MW (2007) Different regional patterns of cortical thinning in Alzheimer's disease and frontotemporal dementia. Brain 130:1159-1166.

Enzinger C, Fazekas F, Matthews PM, Ropele S, Schmidt H, Smith S, Schmidt $R$ (2005) Risk factors for progression of brain atrophy in aging: six-year follow-up of normal subjects. Neurology 64:1704-1711.
Ezekiel F, Chao L, Kornak J, Du AT, Cardenas V, Truran D, Jagust W, Chui H, Miller B, Yaffe K, Schuff N, Weiner M (2004) Comparisons between global and focal brain atrophy rates in normal aging and Alzheimer disease: boundary shift integral versus tracing of the entorhinal cortex and hippocampus. Alzheimer Dis Assoc Disord 18:196-201.

Fennema-Notestine C, Hagler DJ Jr, McEvoy LK, Fleisher AS, Wu EH, Karow DS, Dale AM (2009) Structural MRI biomarkers for preclinical and mild Alzheimer's disease. Hum Brain Mapp 30:3238-3253.

Fischl B, Dale AM (2000) Measuring the thickness of the human cerebral cortex from magnetic resonance images. Proc Natl Acad Sci U S A 97: $11050-11055$.

Fischl B, Sereno MI, Dale AM (1999a) Cortical surface-based analysis. II: inflation, flattening, and a surface-based coordinate system. Neuroimage 9:195-207.

Fischl B, Sereno MI, Tootell RB, Dale AM (1999b) High-resolution intersubject averaging and a coordinate system for the cortical surface. Hum Brain Mapp 8:272-284.

Fischl B, Salat DH, Busa E, Albert M, Dieterich M, Haselgrove C, van der Kouwe A, Killiany R, Kennedy D, Klaveness S, Montillo A, Makris N, Rosen B, Dale AM (2002) Whole brain segmentation: automated labeling of neuroanatomical structures in the human brain. Neuron 33:341-355.

Fischl B, van der Kouwe A, Destrieux C, Halgren E, Ségonne F, Salat DH, Busa E, Seidman LJ, Goldstein J, Kennedy D, Caviness V, Makris N, Rosen B, Dale AM (2004) Automatically parcellating the human cerebral cortex. Cereb Cortex 14:11-22.

Fjell AM, Walhovd KB, Amlien I, Bjørnerud A, Reinvang I, Gjerstad L, Cappelen T, Willoch F, Due-Tønnessen P, Grambaite R, Skinningsrud A, Stenset V, Fladby T (2008) Morphometric changes in the episodic memory network and tau pathologic features correlate with memory performance in patients with mild cognitive impairment. AJNR Am J Neuroradiol 29:1183-1189.

Fjell AM, Westlye LT, Amlien I, Espeseth T, Reinvang I, Raz N, Agartz I, Salat DH, Greve DN, Fischl B, Dale AM, Walhovd KB (2009a) Minute effects of sex on the aging brain: a multi-sample MRI-study of healthy aging and Alzheimer's disease. J Neurosci 29:8774-8783.

Fjell AM, Westlye LT, Amlien I, Espeseth T, Reinvang I, Raz N, Agartz I, Salat DH, Greve DN, Fischl B, Dale AM, Walhovd KB (2009b) High consistency of regional cortical thinning in aging across multiple samples. Cereb Cortex 19:2001-2012.

Folstein MF, Folstein SE, McHugh PR (1975) “Mini-mental state." A practical method for grading the cognitive state of patients for the clinician. J Psychiatr Res 12:189-198.

Fotenos AF, Snyder AZ, Girton LE, Morris JC, Buckner RL (2005) Normative estimates of cross-sectional and longitudinal brain volume decline in aging and AD. Neurology 64:1032-1039.

Freeborough PA, Fox NC (1998) Modeling brain deformations in Alzheimer disease by fluid registration of serial 3D MR images. J Comput Assist Tomogr 22:838-843.

Good CD, Johnsrude IS, Ashburner J, Henson RN, Friston KJ, Frackowiak RS (2001) A voxel-based morphometric study of ageing in 465 normal adult human brains. Neuroimage 14:21-36.

Grandy J (1997) Efficient computation of volume of hexahedral cells. Livermore, CA: Lawrence Livermore National Laboratory.

Greenberg DL, Messer DF, Payne ME, Macfall JR, Provenzale JM, Steffens DC, Krishnan RR (2008) Aging, gender, and the elderly adult brain: an examination of analytical strategies. Neurobiol Aging 29:290-302.

Gunning-Dixon FM, Head D, McQuain J, Acker JD, Raz N (1998) Differential aging of the human striatum: a prospective MR imaging study. AJNR Am J Neuroradiol 19:1501-1507.

Holland D, Hagler DJ, Fennema-Notestine C, Dale AM (2008) PI-249: longitudinal nonlinear registration and quantitative analysis of change in whole brain and regions of interest. Alzheimers Dement 4:T288.

Jack CR Jr, Petersen RC, Xu Y, O’Brien PC, Smith GE, Ivnik RJ, Tangalos EG, Kokmen E (1998) Rate of medial temporal lobe atrophy in typical aging and Alzheimer's disease. Neurology 51:993-999.

Jack CR Jr, Petersen RC, Xu YC, O’Brien PC, Smith GE, Ivnik RJ, Boeve BF, Waring SC, Tangalos EG, Kokmen E (1999) Prediction of AD with MRI-based hippocampal volume in mild cognitive impairment. Neurology 52:1397-1403.

Jernigan TL, Gamst AC (2005) Changes in volume with age-consistency 
and interpretation of observed effects. Neurobiol Aging 26:1271-1274; discussion 1275-1278.

Jernigan TL, Archibald SL, Berhow MT, Sowell ER, Foster DS, Hesselink JR (1991) Cerebral structure on MRI, part I: localization of age-related changes. Biol Psychiatry 29:55-67.

Jernigan TL, Archibald SL, Fennema-Notestine C, Gamst AC, Stout JC, Bonner J, Hesselink JR (2001) Effects of age on tissues and regions of the cerebrum and cerebellum. Neurobiol Aging 22:581-594.

Jovicich J, Czanner S, Greve D, Haley E, van der Kouwe A, Gollub R, Kennedy D, Schmitt F, Brown G, Macfall J, Fischl B, Dale A (2006) Reliability in multi-site structural MRI studies: effects of gradient non-linearity correction on phantom and human data. Neuroimage 30:436-443.

Luft AR, Skalej M, Schulz JB, Welte D, Kolb R, Bürk K, Klockgether T, Voight K (1999) Patterns of age-related shrinkage in cerebellum and brainstem observed in vivo using three-dimensional MRI volumetry. Cereb Cortex 9:712-721.

Makris N, Meyer JW, Bates JF, Yeterian EH, Kennedy DN, Caviness VS (1999) MRI-based topographic parcellation of human cerebral white matter and nuclei II. Rationale and applications with systematics of cerebral connectivity. Neuroimage 9:18-45.

McDonald CR, McEvoy LK, Gharapetian L, Fennema-Notestine C, Hagler DJ Jr, Holland D, Koyama A, Brewer JB, Dale AM (2009) Regional rates of neocortical atrophy from normal aging to early Alzheimer's disease. Neurology 73:457-465.

McEvoy LK, Fennema-Notestine C, Roddey JC, Hagler DJ Jr, Holland D, Karow DS, Pung CJ, Brewer JB, Dale AM (2009) Alzheimer disease: quantitative structural neuroimaging for detection and prediction of clinical and structural changes in mild cognitive impairment. Radiology 251:195-205.

McKhann G, Drachman D, Folstein M, Katzman R, Price D, Stadlan EM (1984) Clinical diagnosis of Alzheimer's disease: report of the NINCDSADRDA Work Group under the auspices of Department of Health and Human Services Task Force on Alzheimer's Disease. Neurology 34:939-944.

Miller MI, Christensen GE, Amit Y, Grenander U (1993) Mathematical textbook of deformable neuroanatomies. Proc Natl Acad Sci U S A 90:11944-11948.

Morris JC (1993) The Clinical Dementia Rating (CDR): current version and scoring rules. Neurology 43:2412-2414.

Pfefferbaum A, Sullivan EV, Rosenbloom MJ, Mathalon DH, Lim KO (1998) A controlled study of cortical gray matter and ventricular changes in alcoholic men over a 5-year interval. Arch Gen Psychiatry 55:905-912.

Raz N, Rodrigue KM (2006) Differential aging of the brain: patterns, cognitive correlates and modifiers. Neurosci Biobehav Rev 30:730-748.

Raz N, Rodrigue KM, Kennedy KM, Dahle C, Head D, Acker JD (2003) Differential age-related changes in the regional metencephalic volumes in humans: a 5-year follow-up. Neurosci Lett 349:163-166.

Raz N, Gunning-Dixon F, Head D, Rodrigue KM, Williamson A, Acker JD (2004) Aging, sexual dimorphism, and hemispheric asymmetry of the cerebral cortex: replicability of regional differences in volume. Neurobiol Aging 25:377-396.

Raz N, Lindenberger U, Rodrigue KM, Kennedy KM, Head D, Williamson A, Dahle C, Gerstorf D, Acker JD (2005) Regional brain changes in aging healthy adults: general trends, individual differences and modifiers. Cereb Cortex 15:1676-1689.

Resnick SM, Goldszal AF, Davatzikos C, Golski S, Kraut MA, Metter EJ, Bryan $\mathrm{RN}$, Zonderman $\mathrm{AB}$ (2000) One-year age changes in MRI brain volumes in older adults. Cereb Cortex 10:464-472.

Resnick SM, Pham DL, Kraut MA, Zonderman AB, Davatzikos C (2003) Longitudinal magnetic resonance imaging studies of older adults: a shrinking brain. J Neurosci 23:3295-3301.

Salat DH, Buckner RL, Snyder AZ, Greve DN, Desikan RS, Busa E, Morris JC, Dale AM, Fischl B (2004) Thinning of the cerebral cortex in aging. Cereb Cortex 14:721-730.

Salat DH, Lee SY, van der Kouwe AJ, Greve DN, Fischl B, Rosas HD (2009) Age-associated alterations in cortical gray and white matter signal intensity and gray to white matter contrast. Neuroimage 48:21-28.

Scahill RI, Schott JM, Stevens JM, Rossor MN, Fox NC (2002) Mapping the evolution of regional atrophy in Alzheimer's disease: unbiased analysis of fluid-registered serial MRI. Proc Natl Acad Sci U S A 99:4703-4707.

Scahill RI, Frost C, Jenkins R, Whitwell JL, Rossor MN, Fox NC (2003) A longitudinal study of brain volume changes in normal aging using serial registered magnetic resonance imaging. Arch Neurol 60:989-994.

Schuff N, Woerner N, Boreta L, Kornfield T, Shaw LM, Trojanowski JQ, Thompson PM, Jack CR Jr, Weiner MW (2009) MRI of hippocampal volume loss in early Alzheimer's disease in relation to ApoE genotype and biomarkers. Brain 132:1067-1077.

Singh V, Chertkow H, Lerch JP, Evans AC, Dorr AE, Kabani NJ (2006) Spatial patterns of cortical thinning in mild cognitive impairment and Alzheimer's disease. Brain 129:2885-2893.

Sled JG, Zijdenbos AP, Evans AC (1998) A nonparametric method for automatic correction of intensity nonuniformity in MRI data. IEEE Trans Med Imaging 17:87-97.

Sullivan EV, Rosenbloom M, Serventi KL, Pfefferbaum A (2004) Effects of age and sex on volumes of the thalamus, pons, and cortex. Neurobiol Aging 25:185-192.

Van Der Werf YD, Tisserand DJ, Visser PJ, Hofman PA, Vuurman E, Uylings HB, Jolles J (2001) Thalamic volume predicts performance on tests of cognitive speed and decreases in healthy aging. A magnetic resonance imaging-based volumetric analysis. Brain Res Cogn Brain Res 11:377-385.

Walhovd KB, Fjell AM, Reinvang I, Lundervold A, Dale AM, Eilertsen DE, Quinn BT, Salat D, Makris N, Fischl B (2005) Effects of age on volumes of cortex, white matter and subcortical structures. Neurobiol Aging 26: 1261-1270; discussion 1275-1278.

Walhovd KB, Westlye LT, Amlien I, Espeseth T, Reinvang I, Raz N, Agartz I, Salat D, Greve D, Fischl B, Dale A (2009) Consistent neuroanatomical age-related volume differences across multiple samples. Neurobiol Aging. Advance online publication. Retrieved June 29, 2009. doi: 10.1016/j.neurobiolaging. 2009.05.013.

Westlye LT, Walhovd KB, Dale AM, Espeseth T, Reinvang I, Raz N, Agartz I, Greve DN, Fischl B, Fjell AM (2009) Increased sensitivity to effects of normal aging and Alzheimer's disease on cortical thickness by adjustment for local variability in gray/white contrast: a multi-sample MRI study. Neuroimage 47:1545-1557. 